\title{
QCD EVOLUTION AND TMD/SPIN EXPERIMENTS
}

\author{
JIAN-PING CHEN \\ Thomas Jefferson National Accelerator Facility, \\ 12000 Jefferson Avenue, \\ Newport News, VA 23606, USA \\ jpchen@jlab.org
}

\begin{abstract}
Transverse Spin and Transverse Momemtum Dependent (TMD) distribution study has been one of the main focuses of hadron physics in recent years. The initial exploratory Semi-Incluisve Deep-Inelastic-Scattering (SIDIS) experiments with transversely polarized proton and deuteron from HERMES and COMPASS attracted great attention and lead to very active efforts in both experiments and theory. QCD factorization has been carefully studied. A SIDIS experiment on the neutron with a polarized ${ }^{3} \mathrm{He}$ target was performed at JLab. Recently published results will be shown. Precision TMD experiments are planned at JLab after the $12 \mathrm{GeV}$ energy upgrade. The approved experiments with a new SoLID spectrometer on both the proton and neutron will be presented. Proper QCD evolution treatments beyond collinear cases become crucial for the precision study of the TMDs. Experimentally, $Q^{2}$ evolution and higher-twist effects are often closely related. The experience of study higher-twist effects in the cases of moments of the spin structure functions will be discussed.
\end{abstract}

Keywords: Spin; transverse; higher-twist.

\section{Introduction}

Spin-dependent observables have been a powerful tool to probe the internal structure of the nucleon and to understand the dynamics of the strong interaction. Experiments involving spin degrees of freedom have lead to numerous surprises, puzzles and discoveries. The so called "spin crisis" in the 1980s revealed the limitation of naive quark-parton models and led to intensive worldwide efforts, both experimental and theoretical, to understand the nucleon spin structure. The nucleon spin structure study has grown from mainly on the longitudinal spin in the last thirty years to recently rapidly increasing interests on the transverse spin. Transverse Spin and Transverse Momentum Dependent (TMD) distribution study has been the focus of hadron physics in recent years. 


\subsection{Transversity and TMD distributions}

The transverse spin (transversity) distributions, $\delta q\left(x, Q^{2}\right)$, are fundamental leadingtwist (twist-2) quark distributions, similar to the unpolarized and polarized parton distributions, $q\left(x, Q^{2}\right)$ and $\Delta q\left(x, Q^{2}\right)$. In quark-parton models, they describe the net transverse polarization of quarks in a transversely polarized nucleon. Several special features of the transversity distributions make them uniquely interesting:

- The difference between the transversity and the longitudinal distributions is purely due to relativistic effects. In the absence of relativistic effects (as in the non-relativistic quark model, where boosts and rotations commute), the transversity distributions are identical to the longitudinally polarized distributions.

- The quark transversity distributions do not mix with gluonic effects ${ }^{1}$ and therefore follow a much simpler evolution and have a valence-like behavior.

- The positivity of helicity amplitudes leads to the Soffer's inequality ${ }^{2}$ for the transversity: $|\delta q| \leq \frac{1}{2}(q+\Delta q)$.

- The lowest moment of $\delta q$ measures a simple local operator analogous to the axial charge, known as the "tensor charge", which can be calculated from lattice QCD.

Due to the chiral-odd nature of the transversity distribution, it cannot be measured in inclusive DIS experiments. In order to measure $\delta q\left(x, Q^{2}\right)$, an additional chiral-odd object is required, such as double-spin asymmetries in Drell-Yan processes, single target-spin azimuthal asymmetries in SIDIS reactions.

In the study of the unpolarized, longitudinally-polarized and transverselypolarized parton distributions, the parton transverse momenta are usually integrated over, so that the distributions depend only on the longitudinal momentum. Over 40-year's study has lead to precision information on the nucleon internal structure, but only in one dimension. The TMD distributions will provide a detailed map of the nucleon structure in 3-D momentum space. Study of TMDs will also allow us to learn about the orbital motion of the quarks and the effects from spin-orbital correlations. Furthermore, it provides a rich source of information on fundamental properties of QCD, such as the color gauge invariance. It will help to unravel the complexity of the quark-gluon interaction and shed light on the dynamics of the color confinement.

The first results of single target spin asymmetries in SIDIS from HERMES ${ }^{3}$ (for the proton) and COMPASS ${ }^{4}$ collaborations (for the deuteron and the proton) showed clear non-zero results for both the Collins and Sivers asymmetries for the proton, offering a first glimpse of possible effects caused by the transversity and TMD distributions. A global fit was performed on these data together with $e^{+} e^{-}$colliding data from Belle. Transversity, Sivers Function and Collins Functions were extracted from the global fit with some assumptions. ${ }^{5}$ The recent results from COMPASS $^{6}$ on the proton confirmed the early results on the Collins asymmetries while the results on the Sivers asymmetries have some difference from those of the 
HERMES. Since these two sets of results are at different $Q^{2}$ values, $Q^{2}$ evolution is needed to have a proper comparison.

\section{Results from JLab E06-010: Single and Double Spin Asymmetries of the Neutron from SIDIS on a Transversely Polarized ${ }^{3} \mathrm{He}$}

The first measurement of target single spin asymmetries for the neutron and ${ }^{3} \mathrm{He}$ was performed in the semi-inclusive ${ }^{3} \mathrm{He}\left(e, e^{\prime} \pi\right) X$ reaction on a transversely polarized target. ${ }^{7}$ The experiment, conducted at Jefferson Lab using a $5.9 \mathrm{GeV}$ electron beam, covers a range of $0.14<x<0.34$ with $1.3<Q^{2}<2.7 \mathrm{GeV}^{2}$. The Collins and Sivers moments were extracted from the azimuthal angular dependence of the measured asymmetries and are shown in Fig. 1. The extracted $\pi$ Collins moments for ${ }^{3} \mathrm{He}$ are consistent with zero, except for the $\pi^{+}$moment at $x=0.34$, which deviates from zero by $2.3 \sigma$. While the $\pi^{-}$Sivers moments are consistent with zero but favor positive values, the $\pi^{+}$Sivers moments are within one $\sigma$ from zero and favor negative values. The neutron results were extracted using the nucleon effective polarization and the measured cross section ratio of proton to ${ }^{3} \mathrm{He}$ and are show in Fig. 2 The Collins moments are compared with the phenomenological fit, ${ }^{5}$ a light-cone quark model calculation ${ }^{8}$ and quark-diquark model calculations. ${ }^{9}$ The phenomenological fit and the model calculations, which assume Soffer's bound, predict rather small Collins asymmetries which are mostly consistent with our data. However, the $\pi^{+}$ Collins moment at $x=0.34$ is suggestive of a noticeably more negative value at the $2 \sigma$ level. Our data favor negative $\pi^{+}$Sivers moments, while the $\pi^{-}$moments

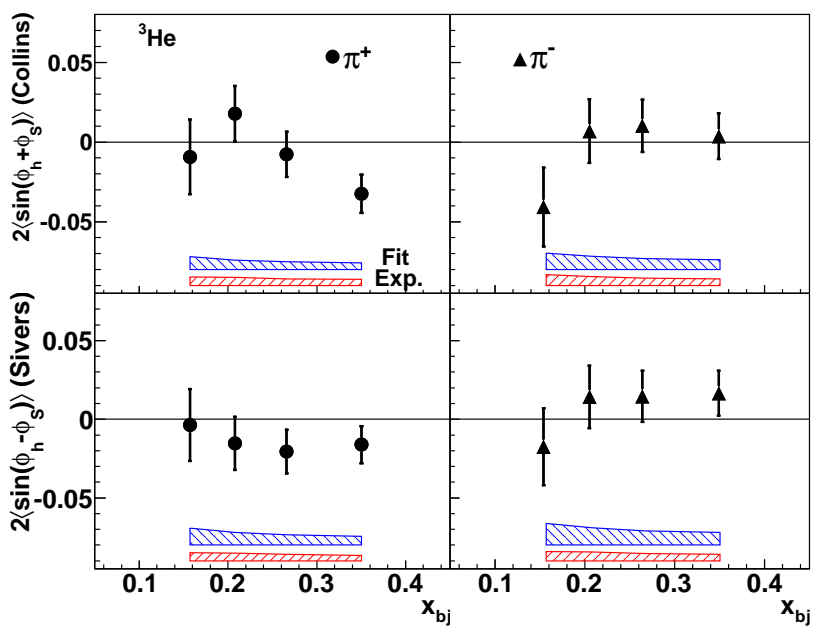

Fig. 1. The extracted Collins/Sivers moments on ${ }^{3}$ He are shown together with uncertainty bands (see text) for both $\pi^{+}$and $\pi^{-}$electro-production. 


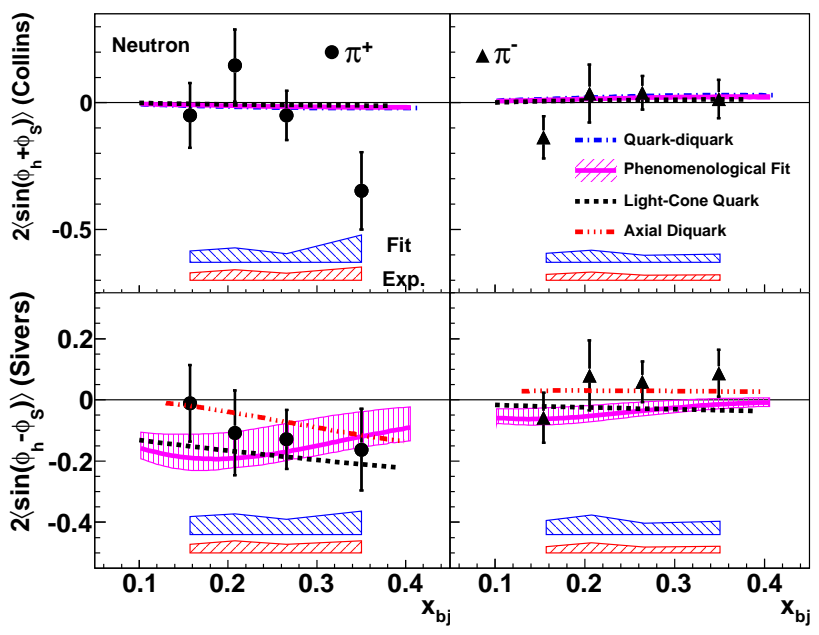

Fig. 2. The extracted Collins/Sivers moments on neutron are shown together with uncertainty bands (see text) for both $\pi^{+}$and $\pi^{-}$electro-production.

are close to zero. Such behavior independently supports a negative $d$-quark Sivers function within the parton model picture, which has been suggested by predictions of the phenomenological fit ${ }^{5}$ to HERMES and COMPASS data, a light-cone quark model calculation, ${ }^{10}$ and an axial diquark model calculation. ${ }^{11}$

These data provide the best current measurement of the neutron Sivers moments in the valence region $(x>0.1)$, and the best neutron Collins moments for $x>0.2$, which will further improve the extraction of $d$-quark distributions in these regions. This experiment has demonstrated the power of polarized ${ }^{3} \mathrm{He}$ as an effective polarized neutron target, and has laid the foundation for future high precision measurements of TMDs with a large acceptance detector SoLID following the JLab $12 \mathrm{GeV}$ upgrade ${ }^{12}$ and at an electron-ion collider. These future SIDIS data taken over a broad range of $Q^{2}$ will also allow an accurate determination of higher twist contribution.

Beam-Target Double Spin Asymmetry $A_{L T}$ on a Transversely Polarized ${ }^{3} \mathrm{He}$ Target was also measured in the same experiment. ${ }^{13}$ The corresponding neutron $A_{L T}$ asymmetries were extracted from the measured ${ }^{3} \mathrm{He}$ asymmetries and proton over ${ }^{3} \mathrm{He}$ cross section ratios using the effective polarization approximation. These new data probe the transverse momentum dependent parton distribution function $g_{1 T}^{q}$ and therefore provide access to quark spin-orbit correlations. Our results indicate a positive azimuthal asymmetry for $\pi^{-}$production on ${ }^{3} \mathrm{He}$ and the neutron, while our $\pi^{+}$asymmetries are consistent with zero.

The extracted $A_{L T}$ results for the neutron and the $A_{L L}$ correction applied to the data are shown in Fig. 3 and are compared to several model calculations, including 


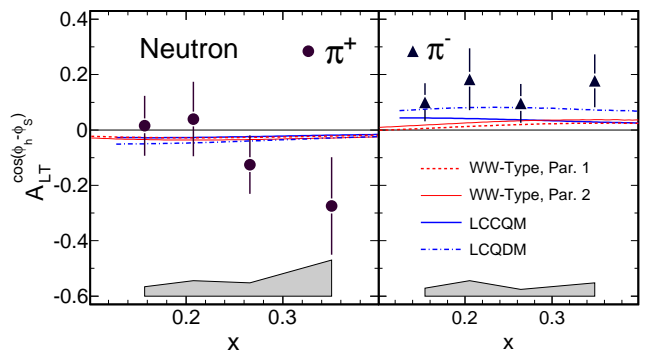

Fig. 3. Neutron azimuthal asymmetry $A_{L T}$ for positive (left) and negative (right) charged pions vs $x$.

WW-type approximations with parametrizations, ${ }^{14}$ a light-cone constituent quark model (LCCQM) ${ }^{15}$ and a light-cone quark-diquark model (LCQDM). ${ }^{16}$ While the extracted $A_{L T}^{n}\left(\pi^{+}\right)$is consistent with zero within the uncertainties, $A_{L T}^{n}\left(\pi^{-}\right)$is consistent in sign with these model predictions but favors a larger magnitude. Sizable asymmetries could be expected for future experiments, including corresponding SIDIS asymmetries on a proton target and the double-polarized asymmetry in DrellYan dilepton production. While the $\pi^{+}$and $\pi^{-}$data are consistent with the interplay between S-P and P-D wave interference terms predicted by the LCCQM and LCQDM models, the magnitude of the measured $\pi^{-}$asymmetry suggests a larger total contribution from such terms than that found in the LCCQM. The larger magnitude of the data compared to the WW-type calculations suggests either a different $P_{T}$ dependence of $A_{L T}$ than assumed in the calculations, a significant role for subleading-twist effects, or both. The statistical precision and kinematic coverage of the present data cannot distinguish between these scenarios. In summary, a positive asymmetry was observed for ${ }^{3} \mathrm{He}\left(e, e^{\prime} \pi\right) X$ and $n\left(e, e^{\prime} \pi\right) X$, providing the first experimental indication of a nonzero $A_{L T}$, which at leading twist leads to a nonzero $g_{1 T}$. When combined with measurements on proton and deuteron targets, these new data will aid the flavor-decomposition of the $g_{1 T}$ TMD PDFs. This work has laid the foundation for the future high-precision mapping of $A_{L T}$ following the JLab 12 GeV upgrade ${ }^{12}$ and at an electron-ion collider, which will provide a comprehensive understanding of the $g_{1 T}$ TMD PDF and the subleading-twist effects.

\section{Planned JLab 12 GeV Program: Precision Study of Transverse Spin and TMD with the SoLID Spectrometer on Both the Proton and Neutron}

With $12 \mathrm{GeV}$ energy upgrade of CEBAF, a new window opens up for precision study of the nucleon transverse spin and transverse momentum dependent distributions. To precisely map out the multi-dimensional (in momentum space) structure, 
both high luminosity and large acceptance spectrometers are necessary. A new spectrometer SoLID (Solenoidal Large Intensity Device) was proposed to be used for precision mapping of the multi-dimensional TMD asymmetries. Three SIDIS experiments were approved by the JLab PACs with the highest rating (A) in the last two years. Two experiments will carry out precision measurements of Single target Spin Asymmetries (SSA) and Double target-beam Spin Asymmetries (DSA) from semi-inclusive electroproduction of charged pions from both a transversely polarized proton and a polarized ${ }^{3} \mathrm{He}$ target in Deep-Inelastic-Scattering kinematics using 11 and $8.8 \mathrm{GeV}$ electron beams. One experiment will be made on longitudinally polarized ${ }^{3} \mathrm{He}$ target. The full $2 \pi$ azimuthal angular coverage is essential in controlling the systematic uncertainties in extracting different asymmetries. The proposed experiments will provide precise $4-\mathrm{D}\left(x, z, P_{T}\right.$ and $\left.Q^{2}\right)$ data on the Collins, Sivers, pretzelosity and Worm-Gear asymmetries for the proton and the neutron through the azimuthal angular dependence. The results from these experiments, when combined with the future proton Collins asymmetry measurement and the Collins fragmentation function determined from the $e^{+} e^{-}$collision data, will allow for a flavor separation of the quark tensor charge, and achieve a determination of the tensor charge of $\mathrm{u}$ and $\mathrm{d}$ quark to better than 10\%. The extracted Sivers, pretzelosity and Worm-Gear asymmetries will provide important information to understand the correlation between the quark orbital angular momentum and the nucleon spin.

The projected results for $\pi^{+}$Collins asymmetry at one typical kinematic bin, $0.45>z>0.4,3>Q^{2}>2$ is shown in Fig. 4. The x-axis corresponds to the Bjorken $x$. The y-axis on the left side is $P_{T}$ which is the transverse momentum. The $y$-axis on the right side shows the scale of the asymmetry. The y-position of the projections shows the average $P_{T}$ value for the corresponding kinematic bin. The statistical uncertainties follow the scale on the right side of $y$-axis. The scale of the theoretical calculations follow the right side y-axis. Also shown in the figure are theoretical predictions from Anselmino et al. [5], Ma et al. [9], Pasquini et al. [15] and Vogelsang and Yuan [17] for the Collins asymmetry. Complete projections for $\pi^{+}\left(\pi^{-}\right)$Collins/pretzelosity/Sivers asymmetries in terms of 4-D ( $x, z, P_{T}$ and $\left.Q^{2}\right)$ kinematic bins can be found ain Ref. [12], and the kinematic range covered is $8 \mathrm{GeV}^{2}>Q^{2}>1 \mathrm{GeV}^{2}, 0.7>z>0.3,1.6 \mathrm{GeV}>P_{T}>0$, and $0.65>x>0.05$.

These data will allow us to extract TMDs in the valence quark region with the ultimate precision. A future Electron-Ion Collider would allow precision study be expanded to the sea quarks and the gluons.

\section{Higher-Twist Study with Moments of Spin Structure Functions}

The high precision study of TMDs requires the understanding the $Q^{2}$ dependence. The $Q^{2}$ dependence comes from the $Q^{2}$ evolution and the higher-twist efffects. To preperly study $Q^{2}$ evolution, higher-twist effects often cannot be neglected.

In the unpolarized and longitudinal spin (helicity) cases, higher-twist effects were studied in certain limited situations. Here I will give a description of a study using 


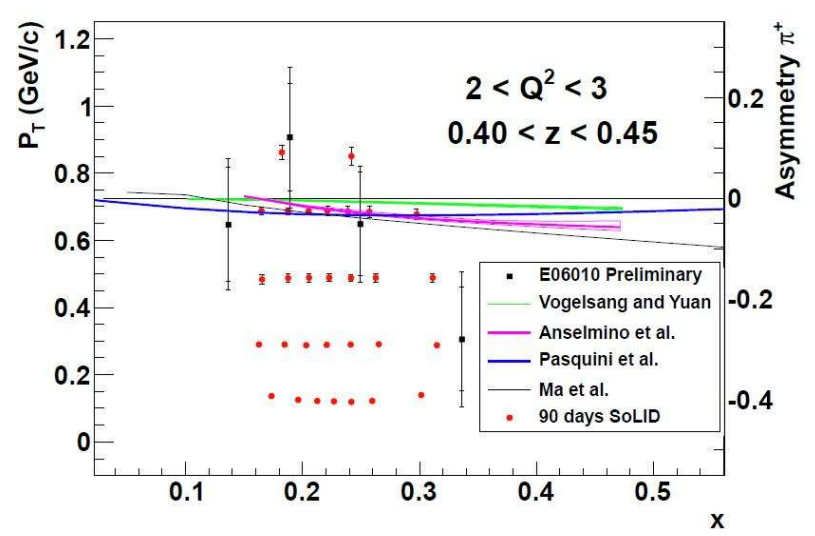

Fig. 4. $12 \mathrm{GeV}$ Projections with SoLID and a transversely polarized ${ }^{3} \mathrm{He}$ target for $\pi^{+}$Collins asymmetries at $0.45>z>0.4,3>Q^{2}>2$.

the moments of the spin structure functions $g_{1}$ and $g_{2}[18,19]$. The leading-twist (twist-2) part of the $g_{1}$ moment provides the information on the contribution of the quark spin to the nucleon spin. The 2 nd moment $d_{2}\left(Q^{2}\right)$, which is the moment of $g_{2}$ subtracting the lead-twist part $\left(g_{2}^{W W}\right.$, which can be obtained from the $g_{1}$ contribution through the Wandzura and Wilczek relation), provides a clean measure of the twist- 3 matrix element $d_{2}$. Several experiments have measured $d_{2}\left(Q^{2}\right)$ and future $12 \mathrm{GeV}$ measurement is planned. We also performed an extraction of the twist- 4 term from the $g_{1}$ moment and the twist- 4 matrix element, $f_{2}$, was extracted. The matrix elements $d_{2}$ and $f_{2}$ are the so called "color polarizabilities" or "Lorentz Forces" which are fundamental property of the nucleon. They can be calculated with Lattice QCD and theory models. The matrix element $d_{2}$ has been calculated with both LQCD and models. It provides a bench test of LQCD calculations since both LQCD and experiments can reach high precision for this quantity.

\section{Summary}

Transverse spin and TMDs are one of the main focuses in hadron physics. Exploratory experiments already show many interest features. Results from a recent JLab experiment on the neutron with a transversely polarized ${ }^{3} \mathrm{He}$ target are discussed. Precision experiments after JLab $12 \mathrm{GeV}$ upgrade and with a new proposed large acceptance spectrometer, SoLID, are presented. High-precision study of TMDs will require preper treatment of $Q^{2}$ dependence. To study $Q^{2}$ evolution, higher-twist effects also need to be considered. A higher-twist study in the longitudinal spin cases is presented.

\section{References}

1. C. Bourrely, J. Soffer and O. V. Teryaev, Phys. Lett. B420, 375 (1998). 
2. J. Soffer, Phys. Rev. Lett. 74, 1292 (1995).

3. A. Airapetian, et al., Phys. Rev. Lett. 94, 012002 (2005).

4. The COMPASS collaboration, Phys. Rev. Lett. 94, 202002 (2005); Nucl. Phys. B765, 31-70 (2007).

5. M. Anselmino et al.,Phys. Rev. D72, 094007 (2005); M. Anselmino et al., Phys. Rev. D75, 054032 (2007).

6. The COMPASS collaboration, Phys. Lett. B717, 376 (2012); Phys. Lett. B717, 383 (2012).

7. JLab E06-010 Collaboration, X. Qian et al.,Phys. Rev. Lett. 107, 072003 (2011).

8. S. Boffi et al.,Phys. Rev. D79, 094012 (2009); B. Pasquini et al., Phys. Rev. D78, 034025 (2008).

9. J. She and B. Q. Ma, Phys. Rev. D83, 037502 (2011).

10. B. Pasquini and P. Schweitzer, arXiv:1103.5977 (2011).

11. L. P. Gamberg et al., Phys. Rev. D77, 094016 (2008).

12. JLab E12-10-006, Spokespersons: J. P. Chen, H. Gao, X. Jiang, X. Qian and J. C. Peng. JLab E12-11-007, Spokespersons: J. P. Chen, J. Huang, Y. Qiang and W. Yan. JLab E12-11-108, Spokespersons: K. Allada, J. P. Chen, H. Gao, X. Li and Z. E. Meziani.

13. JLab E06-010 Collaboration, J. Huang et al.,Phys. Rev. Lett. 108, 052001 (2012).

14. A. Kotzinian, B. Parsamyan, and A. Prokudin, Phys. Rev. D73, 114017 (2006).

15. S. Boffi, A. V. Efremov, B. Pasquini, and P. Schweitzer, Phys. Rev. D79, 094012 (2009); B. Pasquini, S. Cazzaniga, and S. Boffi, Phys. Rev. D78, 034025 (2008).

16. J. Zhu and B.-Q. Ma, Phys. Lett. B696, 246 (2011).

17. W. Vogelsang and F. Yuan, private communications.

18. J. P. Chen, Int. J. Mod. Phys. E19 1893 (2010).

19. S. E. Kuhn, J. P. Chen and E. Leader, Prog. Part. Nucl. Phys. 63 1, (2009). 